\title{
Using Behavioral Economics to Promote HIV Prevention for Key Populations
}

\author{
Sebastian Linnemayr ${ }^{1}$, Sarah MacCarthy ${ }^{1 *}$, Zachary Wagner ${ }^{1}$, Joanna L. Barreras ${ }^{2}$ and Frank H. Galvan ${ }^{2}$ \\ ${ }^{1} R A N D$ Corporation, Santa Monica, USA
}

${ }^{2}$ Bienestar Human Services, Inc. Los Angeles, USA

\begin{abstract}
In this short communication, we discuss some key behavioral economic (BE) biases that likely minimize HIV prevention efforts, explore why certain key populations such as men who have sex with men or transgender womenmay be more likely to succumb to these biases, and suggest how incentives informed by BE can support these populations in their effort to remain HIV-negative. Based on our formative work in an ongoing study, we discuss two important insights regarding the use of incentives to inform future HIV prevention efforts. First, participants often expressed more excitement for prizes that were viewed as fun (e.g., movie gift cards) or luxurious (e.g., cosmetics gift cards) rather than necessities (e.g., grocery store gift cards) of the same financial value and suggests that including an element of fun can be a powerful tool for incentivizing safe HIV-related behavior. Second, participants preferred not to be "paid" to display health behaviors, indicating the way incentives are given out (and perceived) is central to their success. Going forward, a BE perspective can help improve the impact of incentives - and increase their costeffectiveness by carefully adapting them to the preferences of their recipients.
\end{abstract}

Keywords: Behavioral economics; HIV prevention; key populations

\section{Introduction}

The decision-making errors or behavioral "biases" highlighted by behavioral economics (BE) are particularly important for health. For example, they may cause individuals to overeat or smoke even when they realize that these activities are unhealthy and are, in fact, highly motivated to avoid them $[1,2]$. Such biases may also act as barriers for people attempting to remain HIV-negative and may be stronger for key populations [3]. Behavioral biases might hinder the effectiveness of traditional HIV prevention interventions, and recognizing the decision-making errors relevant for vulnerable populations provides an opportunity to develop novel BE interventions to prevent HIV infection $[4,5]$. In this short communication, we discuss what key BE biases likely threaten HIV prevention activities, why key populations such as men who have sex with men (MSM) or transgender women are particularly likely to succumb to these biases, and based on our collective experience informed by preliminary findings from our ongoing study, suggest how BE-based incentives can support these populations in their effort to remain HIV-negative.

\section{Behavioral Biases that are Relevant for HIV Prevention among Key Populations}

Present bias-when people are heavily influenced by short-term considerations at the expense of long-term benefits [6] — is particularly relevant for HIV prevention [4]. The costs of behaviors that prevent HIV infection (such as pre-exposure prophylaxis - PrEP [7], male circumcision, and foregoing the pleasure of unprotected sex) occur immediately, but their benefits are realized in the distant future (in the form of improved life expectancy and life quality) [4]. Another potential barrier to HIV prevention highlighted by BE is limited attention or lack of salience-the concept that people tend to focus their energy on things that are at the forefront of their minds. Individuals may be initially motivated to prevent HIV infection, but because remaining in a healthy state is not very noticeable, over time they may "forget" about the threat of acquiring the disease as other, more pressing concerns shape their behavior.
Both present bias and lack of salience are particularly relevant for key populations at high risk for HIV. Cognitive biases tend to be stronger when stress levels are higher, and the conditions that place someone at high-risk of HIV infection often induce stress [8-10]. Transgender women, who often experience high levels of stress due to constant experiences with discrimination [11], can have more salient concerns than adhering to HIV prevention strategies such as PrEP. For example, accessing PrEP requires engaging with formal healthcare systems to get a prescription for its use. As many transgender women experience discrimination based on their gender identity [e.g., (un)intentional misuse of pronouns, verbal harassment, physical or sexual assault][12-14], transgender women may be more likely to avoid perceived authority figures such as providers, fearing continued negative interactions, and therefore forgo initiating PrEP. The fact that vulnerable populations such as transgender women may be more sensitive to behavioral biases underscores the need to incorporate $\mathrm{BE}$ into interventions designed to mitigate HIV risk among these populations.

\section{$\mathrm{BE}$ interventions to overcome behavioral biases}

Incentives are a common approach for overcoming present bias. Incentives (or taxes) in the present, based on engaging (or not) in the desired behavior, could help make the behavior "worth it" (or not "worth it"). Recent studies have found that incentives rooted in BE can promote HIV testing [15,16], male circumcision [5], sexual practices that decrease risk for HIV $[17,18]$, and treatment adherence

Corresponding author: Sarah MacCarthy, $S c D$, Behavioral and Policy Sciences, RAND Corporation, 1776 Main Street, Santa Monica, CA USA, Tel: 3103930411 (Etxn) 6743; Fax: (310) 260 8175; E-mail: sarahm@rand.org

Received October 24, 2018; Accepted November 26, 2018; Published November 30, 2018

Citation: Linnemayr S, MacCarthy S, Wagner Z, Barreras JL, Galvan FH (2018) Using Behavioral Economics to Promote HIV Prevention for Key Populations. J AIDS Clin Res 9: 780. doi: 10.4172/2155-6113.1000780

Copyright: (C) 2018 Linnemayr S, et al. This is an open-access article distributed under the terms of the Creative Commons Attribution License, which permits unrestricted use, distribution, and reproduction in any medium, provided the original author and source are credited. 
[19]. However, this literature is nascent and there is substantial room for innovation. Cash transfers to overcome structural barriers to HIV prevention are often expensive and have had mixed results [20,21], but cash transfers designed to overcome present bias require much smaller monetary value (only enough to make the behavior worth it, not to provide a living) and therefore are more easily scaled. Some incentives informed by $\mathrm{BE}$ have been shown to be cost effective [22,23]. BE suggests that the effectiveness of incentives depends not only on their size, but also on how they are provided (e.g., more frequent incentives are more effective) [6]. Thus, it is important to understand what incentives the target populations care about and then how best to get the them the desired incentives.

Overcoming limited attention involves consistently bringing the desired behavior to the front of the mind by making it more salient [4]. Few studies have tested interventions designed to make HIV prevention or treatment more salient. One study found that repeated HIV testing among serodiscordant couples made safe sexual behavior more salient by continuing to show the HIV-negative partner that he/she was still HIV negative (i.e., safe sex was still worth the cost) [24]. Text message campaigns that acted as constant reminders to adhere to antiretroviral therapy (ART) have had mixed results $[25,26]$. Such campaigns should be explored further among key populations, potentially as a way to make PrEP use more salient. The financial incentives studies described above could also help make preventive behaviors more salient because the incentive focuses the mind on the desired behavior. Future research should test interventions that make safer sexual behavior, HIV testing, and PrEP adherence consistently salient key populations over time.

\section{Designing BE interventions for key populations}

The $\mathrm{BE}$ insights discussed above highlight that the salience of incentives and the way in which incentives are delivered plays a key role in changing behavior. Thus, it is important to directly ask key populations what incentives matter to them, in order to design an incentives program that will elicit the biggest behavioral response. Our formative work in an ongoing study generated two important insights that helped shape our subsequent HIV prevention intervention [27]. First, we found that Latino men who have sex with men and Latina transgender women expressed more excitement for prizes that were viewed as fun (e.g., movie gift cards) or luxurious (e.g., cosmetics gift cards) rather than necessities (e.g., grocery store gift cards) of the same financial value. Therefore, fun prizes are likely to be more salient for these populations. This finding runs counter to our initial expectations that relatively resource-poor individuals would prefer "practical" prizes that increase their disposable income. However, the finding is consistent with prior work suggesting that including an element of fun can be a powerful tool for incentivizing safe HIV-related behavior partly due to the pleasure derived from games of chance $[19,28,29]$. One interpretation is that the possibility of winning prizes made engaging with HIV prevention strategies more fun. In response, in subsequent work we varied the type of small gift certificates offered to participants, ensuring that we included a range of enjoyable, 'fun' rewards.

A second key finding from our formative work is that participants expressed concern about "getting paid" for engaging in healthy behaviors that they already felt intrinsically motivated to pursue. Indeed, if participants perceive incentives as condescending or paternalistic, the incentives may actually reduce the desired outcome. This is consistent with a $\mathrm{BE}$ insight, which highlights that incentives can crowd out intrinsic motivation [30]. This finding underscores a critical observation from BE: the way incentives are provided matters.
Heeding this advice includes an often-overlooked BE insight that can be used to design incentives: the importance of framing [31]. In our study, this meant dedicating time during the consent process to ensure that participants knew we appreciated their desire to live healthy lives. However, we also conveyed that maintaining healthy behaviors is challenging. The small incentive nudges provided as part of the study were to support the motivation they already had to maintain their HIVnegative status.

\section{Conclusion}

Our findings highlight some of the ways in which a BE perspective can bolster the impact of HIV prevention strategies, especially among key populations, who might be more sensitive to behavioral biases. In particular, this work underscores why researchers must learn directly from the populations themselves what drives their behavior so that $\mathrm{BE}$ interventions can be harnessed to achieve prevention goals. Our ongoing study will provide empirical evidence about the effectiveness of these strategically crafted $\mathrm{BE}$ incentives. In the meantime, our preliminary qualitative findings underscore the importance of balancing the need to keep participants engaged, while also acknowledging their genuine motivations they may already have to improve their health.

\section{Acknowledgement}

This study was supported by R34MH096609. We thank Mary Vaiana for her careful review of the manuscript. We also thank Melina Fuentes for assistance with manuscript preparation.

\section{References}

1. Bramley D, Riddell T, Whittaker R, Corbett T, Lin RB, et al. (2005) Smoking cessation using mobile phone text messaging is as effective in Maori as nonMaori. NZ Med J 118: U1494.

2. Marteau TM, Ashcroft RE, Oliver A (2009) Using financial incentives to achieve healthy behaviour. BMJ p: 338.

3. Linnemayr S, Rice T (2016) Insights from behavioral economics to design more effective incentives for improving chronic health behaviors, with an application to adherence to antiretrovirals. J Acquir Immune Defic Syndr 72: e50-e52.

4. Linnemayr S (2015) HIV prevention through the lens of behavioral economics J Acquir Immune Defic Syndr 68: e61.

5. Thirumurthy H, Masters SH, Rao S, Bronson MA, Lanham M, et al. (2014) Effect of providing conditional economic compensation on uptake of voluntary medical male circumcision in Kenya: A randomized clinical trial. JAMA 312: 703-711.

6. White JS, Dow WH (2015) Intertemporal choices for health. Behavioral Economics and Public Health p: 62

7. Centers for Disease Control and Prevention. Pre-Exposure Prophylaxis (PrEP) 2017.

8. Haushofer J, Fehr E (2014) On the psychology of poverty. Science 344: 862867.

9. Duflo E, Kremer M, Robinson J (2011) Nudging farmers to use fertilizer: Theory and experimental evidence from Kenya. Am Econ Rev 101: 2350-2390.

10. Mani A, Mullainathan S, Shafir E, Zhao J (2013) Poverty impedes cognitive function. Science 341: 976-980.

11. James SE, Herman JL, Rankin S, Keisling M, Mottet L, et al. (2016) The report of the 2015 US transgender survey, Washington, DC

12. James SE, Herman JL, Rankin S, Keisling M, Mottet L, et al. (2015) The report of the 2015 US transgender survey. National Center for Transgender Equality, Washington, DC

13. Rodriguez A, Agardh A, Asamoah BO (2017) Self-reported discrimination in health-care settings based on recognizability as transgender: A cross-sectional study among transgender US citizens. Arch Sex Behav 2017: 1-13.

14. Cruz TM (2014) Assessing access to care for transgender and gender 
Citation: Linnemayr S, MacCarthy S, Wagner Z, Barreras JL, Galvan FH (2018) Using Behavioral Economics to Promote HIV Prevention for Key Populations. J AIDS Clin Res 9: 780. doi: 10.4172/2155-6113.1000780

nonconforming people: A consideration of diversity in combating discrimination. Soc Sci Med 110: 65-73.

15. Thornton RL (2008) The demand for and impact of learning HIV status. Am Econ Rev 98: 1829-1863.

16. Montoy JCC, Dow WH, Kaplan BC (2018) Cash incentives versus defaults for HIV testing: A randomized clinical trial. PloS One 13: e0199833.

17. De-Walque D, Dow WH, Nathan R, Abdul R, Abilahi F, et al. (2012) Incentivising safe sex: A randomised trial of conditional cash transfers for HIV and sexually transmitted infection prevention in rural Tanzania. BMJ Open 2: e000747.

18. Packel LJ, De-Walque D, Feeney KC, Balampama MP, Cooper JE, et al. (2018) A randomized trial of cash incentives for sexual behavior change among female sex workers in Dar es Salaam. Soc Sci Med.

19. Linnemayr S, Stecher C, Mukasa B (2017) Behavioral economic incentives to improve adherence to antiretroviral medication. AIDS 31: 719-726.

20. Baird SJ, Garfein RS, McIntosh CT, Özler B (2012) Effect of a cash transfer programme for schooling on prevalence of HIV and herpes simplex type 2 in Malawi: A cluster randomised trial. The Lancet 379: 1320-1329.

21. Mills EJ, Adhvaryu A, Jakiela P, Birungi J, Okoboi S, et al. (2018) Unconditiona cash transfers for clinical and economic outcomes among HIV-affected Ugandan households. AIDS 32: 2023-2031.

22. Benartzi S, Beshears J, Milkman KL, Sunstein CR, Thaler RH, et al. (2017) Should governments invest more in nudging?. Psychol Sci 28: 1041-1055.

23. Gong CL, Zangwill KM, Hay JW, Meeker D, Doctor JN (2018) Behaviora economics interventions to improve outpatient antibiotic prescribing for acute respiratory infections: A cost-effectiveness analysis. J Gen Intern Med pp: 1-9.
24. Delavande A, Wagner Z, Sood N (2016) The impact of repeat HIV testing on risky sexual behavior: Evidence from a randomized controlled trial in Malawi. $J$ AIDS Clin Res p: 7.

25. Linnemayr S, Huang $H$, Luoto J, Kambugu A, Thirumurthy $H$, et al. (2017) Text messaging for improving antiretroviral therapy adherence: No effects after 1 year in a randomized controlled trial among adolescents and young adults. Am J Public Health 107: 1944-1950.

26. Kelly JD, Giordano TP (2011) Mobile phone technologies improve adherence to antiretroviral treatment in a resource-limited setting: A randomized controlled trial of text message reminders. AIDS 25: 1137.

27. Linnemayr S, MacCarthy S, Kim A, Giguere R, Carballo-Dieguez A, et al. (2018) Behavioral economics-based incentives supported by mobile technology on HIV knowledge and testing frequency among Latino/a men who have sex with men and transgender women: Protocol for a randomized pilot study to test intervention feasibility and acceptability. Trials 19: 540 .

28. Kahneman D, Trversky A (1979) Prospect theory: An analysis of decision under risk. Econometrica 47: 263-291.

29. Nyqvist MB, Corno L, De-Walque D, Svensson J (2015) Using lotteries to incentivize safer sexual behavior: Evidence from a randomized controlled trial on HIV prevention: The World Bank.

30. Kamenica E (2012) Behavioral economics and psychology of incentives. Annu Rev Econ 4: 427-452.

31. Kahneman D (2003) Maps of bounded rationality: Psychology for behavioral economics. American Economic Review 93: 1449-1475. 